\title{
Cerdas de cría a campo abierto en la zona tropical
}

\author{
Breeding sows open field in the tropical zone \\ Zuluaga Ruiz Oscar Javier ${ }^{1}$ y Ocampo Duran Álvaro² \\ ${ }^{1}$ Médico Veterinario Zootecnista y \\ 2Zootecnista, MSc, PhD. Docente de la Universidad de los Llanos \\ aocampo@unillanos.eu.co
}

Recibido 20 de Enero 2016, Aceptado 29 de Abril 2016

\section{RESUMEN}

Se evaluó el comportamiento etológico de seis cerdas de cría (mestizas Landrace $x$ Pietrain) bajo el sistema de producción a campo abierto utilizando una densidad de $450 \mathrm{~m}^{2} /$ cerda en las dos temporadas del año (seca y lluviosa), con el objeto de determinar sus características etológicas. Observándose en principio adaptabilidad, reconocimiento, comportamiento individual y grupal, respuesta frente a estímulos de peligro o amenaza, selección de alimento, horas y áreas de descanso, hozado, y zonas sucias o de excreción. También se observó el comportamiento maternal, con el fin de determinar características etológicas en el sistema a campo abierto. Se llevó un registro diario de parámetros medioambientales como temperaturas máximas y mínimas (de 7:00 am a 5:00 pm), así como la precipitación, para determinar el impacto de estas condiciones en las cerdas. La alimentación de los animales se basó en una dieta balanceada elaborada en el mismo sitio, $2 \mathrm{~kg} / \mathrm{cerda} /$ día y $200 \mathrm{~g}$ adicionales por lechón en animales lactantes. El alimento fue suministrado de manera restringida en dos raciones al día (7:30 a.m. y 4:30 p.m.). El manejo reproductivo se fundamentó en el uso de un reproductor comercial (Landrace $x$ Pietran), realizando el servicio una vez se identificaba el celo. Para establecer la diversidad, cobertura y grado de dominancia de las especies vegetales se realizaron aforos. Las acciones de consumo de agua, pastoreo, exploración, descanso, bañarse en la piscina y pelar, fueron altamente influenciadas por la época del año $(P<0.001)$, también se evidenciaron cambios de actividades de los lotes en relación con los cambios de 
temperatura ambiental registrados. El comportamiento de las cerdas sugiere una interesante capacidad de adaptación a las condiciones de temperatura, precipitación y características del terreno (drenaje), así como al sistema de manejo. Aunque existen coincidencias con los reportes de literatura, se requiere profundizar sobre el comportamiento animal en las condiciones de la Orinoquia y su dinámica de precipitación, radiación solar y temperatura ambiental, para el diseño de instalaciones apropiadas que garanticen condiciones de confort y estrategias de manejo.

Palabras clave: Lechones, etología, pastoreo.

\section{ABSTRACT}

The ethological behavior of six sows was evaluated (Crossbred Landrace $x$ Pietrain) under the system of open field production using a density of $450 \mathrm{~m}^{2} / \mathrm{sow}$ in the two seasons of the year (dry and rainy), in order to determine its behavioral characteristics. Adaptability observed in principle, recognition, individual and group behavior, response to stimuli of danger or threat, food selection, hours and rest areas, rooting, or excretion and dry areas. Maternal behavior was also observed, in order to determine ethological features in the open field system. A daily record of environmental parameters was carried as maximum and minimum temperatures (from 7:00 am to 5:00 pm), as well as precipitation, to determine the impact of these conditions in the sows. Feeding the animals it was based on a balanced diet made on the same site, $2 \mathrm{~kg} / \mathrm{sow} /$ day and $200 \mathrm{~g}$ additional per piglet in lactating animals. The food was supplied narrowly in two servings a day (7:30 a.m. and 4:30 p.m.). The reproductive management was based on the use of a commercial player (Landrace $x$ Pietran), performing the service once identified the zeal. To establish the diversity, coverage and degree of dominance of plant species appraisals were conducted. Shares of water consumption, grazing, exploration, rest, swim in the pool and fighting were highly influenced by the time of year $(P<0.001)$, changes batch activities regarding changes recorded ambient temperature were also evident. The behavior of the sows suggests an interesting ability to adapt to the conditions of temperature, precipitation and soil characteristics (drainage), as well 
as the management system. Although there are coincidences with literature reports, required further animal behavior in the conditions of the Orinoquía and its dynamics of precipitation, solar radiation and ambient temperature for the design of appropriate facilities to ensure comfort conditions and management strategies.

Keywords: Piglets, ethology, grazing.

\section{RESUMO}

O comportamento etológico de seis porcas foi avaliada (Mestiços Landrace $x$ Pietrain) sob o sistema de produção em campo aberto usando uma densidade de $450 \mathrm{~m}^{2} /$ porca em da duas estações do ano (seca e chuvosa), a fim de determinar as suas características de comportamento. Em princípio observa-se adaptabilidade, reconhecimento, comportamento individual e em grupo, resposta a estímulos de perigo ou ameaça, seleção de alimentos, horas e áreas de descanso, hozado, e áreas sujas ou excreção. Também observou-se o comportamento maternal, a fim de determinar características etológicas no sistema de campo aberto. Foi realizada um registro diário de parâmetros ambientais como temperaturas máximas e mínimas (das 7:00 às 5:00 da tarde), bem como precipitação, para determinar o impacto dessas condições sobre as cerdas. Alimentação dos animais foi baseado em uma dieta equilibrada feita no mesmo sítio, $2 \mathrm{~kg} /$ porca/dia e $200 \mathrm{~g}$ adicionais por leitão em animais em lactação. A comida foi fornecida por pouco em duas porções por dia (7:30 am. e 4:30 pm.). Manejo reprodutivo baseou-se no uso de um leitor comercial (Landrace $\mathrm{x}$ Pietran) execução do serviço, uma vez identificadas zelo. Para estabelecer a diversidade, cobertura e grau de domínio das espécies de plantas avaliações foram conduzidas. Ações de consumo de água, pastagem, exploração, descanso, nadar na piscina e briga, foram altamente influenciados pela época do ano $(P<0.001)$. Alterações atividades de lote em relação às mudanças registadas temperatura ambiente também foram evidentes. O comportamento das cerdas sugere uma capacidade interessante de adaptação às condições de temperatura, precipitação e as características do solo (drenagem), bem como o sistema de gestão. Embora haja semelhanças com relatos da literatura, se requer ainda o comportamento 
animal nas condições do Orinoco e sua dinâmica de precipitação, radiação solar e temperatura ambiente para a concepção de instalações adequadas para assegurar condições de conforto e estratégias de gestão.

Palavras-chave: Leitões, etologia, pastagens.

\section{INTRODUCCIÓN}

En Colombia, existe escasa información sobre los parámetros productivos e impacto ambiental del sistema de producción de cerdos a campo abierto. Una de las características fundamentales es que el estiércol porcino hace un gran aporte de materia orgánica, el cual mejora las condiciones físicas, estructura, capacidad de retención de humedad, aporte de nitrógeno e incremento en la población microbiana del suelo (Gomero y Velázquez, 1999). Estas características promueven la lenta liberación de minerales, lo cual ofrece beneficios productivos residuales a largo plazo; convirtiéndose de esta manera, en una alternativa para mitigar el impacto ambiental generado por las explotaciones porcícolas.

En este mismo sentido, en el país la cría de cerdos al aire libre ha sido asociada tradicionalmente a esquemas de manejo en áreas de bosque y de traspatio en zonas periurbanas, principalmente en las regiones del Pacifico y de los Llanos Orientales (Sarria et al., 2001). Este sistema se originó con la llegada de los primeros animales de origen ibérico traídos por los colonizadores para la producción de carne y grasa; generando las razas criollas actuales por cruzamiento y selección, las cuales se han adaptado exitosamente a las condiciones ambientales de Colombia (Cardozo et al., 2010).

Además en los sistemas productivos de cerdos es importante el bienestar animal, que es la manifestación de confort y ausencia de sufrimiento o estrés, que mantiene en equilibrio el estado fisiológico del animal frente a su entorno (Muñoz, 2002). En su ambiente natural el animal expresa costumbres y actividades propias de la especie como si estuviera en total libertad. El sistema de producción de cerdos a campo abierto ofrece en gran medida un estado saludable que minimiza 
las situaciones de estrés favoreciendo al productor mayores índices de rentabilidad.

Rudine et al., (2007) establecieron que los cerdos manejados bajo este sistema expresan en mayor proporción su comportamiento natural, lo cual redujo el estrés, mejoró el estatus sanitario y su inmunidad contra bacterias. Adicionalmente Muriel et al., (2002) reportaron que un mayor bienestar animal asociado al sistema promueve la calidad del producto final, por incremento de los ácidos grasos poliinsaturados (PUFA) $\omega-3$ y $\omega-6$, de lípidos neutros (ácidos grasos de más de 12 $\mathrm{C}$, acilgliceroles, esteroles y sus ésteres, carotenoides, y otros) de lípidos polares (glicerofosfolípidos, glicosilglicerolípidos, esfingofosfolípidos, esfingoglicolípidos); lo cual genera un sabor superior en la carne y consecuentemente beneficios para la salud de los seres humanos.

La tecnificación de los sistemas de producción porcina rompió con el balance o estructura natural-social de los animales al imponer condiciones físicas extremas, con el fin de obtener mejores índices productivos en menor tiempo, llevando al animal a sufrir modificaciones morfofisioetológicas empobreciendo su bienestar, además provocando mayor hacinamiento con la consecuente aparición de comportamientos estereotipados en los individuos conduciendo muchas veces a trastornos de comportamiento irremediables, que repercuten en la deseada rentabilidad (Muñoz, 2002). El sistema a campo abierto en la producción porcina surge como una alternativa para los medianos y pequeños productores bajo la primicia de obtener mejores o iguales utilidades a menor costo en comparación con un sistema tecnificado que requiere gran capital. Además ofrece la posibilidad a los animales de estar en un ambiente natural con la consecuente reducción en la incidencia de problemas en su conducta. Conocer el patrón de actividades de los cerdos constituye una herramienta fundamental para obtener buenos rendimientos productivos y ofrecer una mejor calidad de vida a los animales domésticos que sirven al hombre como fuente de alimento (Campiño y Ocampo, 2010).

Rodarte et al., (2001) establecieron que el comportamiento de los cerdos domésticos es muy similar al de los cerdos silvestres, gracias a su capacidad de 
adaptación, aprendizaje y memoria, lo cual permite que estos cerdos se pueden adaptar satisfactoriamente a los sistemas a campo. Sin embargo, es necesario considerar que en los cerdos las condiciones medioambientales tienen gran influencia sobre el bienestar y comportamiento. Algunos estudios han demostrado que la práctica de permanecer inmóvil durante largos periodos de tiempo en zonas de sombra y, de revolcarse en el lodo en épocas cálidas, son comportamientos instintivos de protección contra la hipertermia, quemaduras solares y golpe de calor.

En cerdos en crecimiento Andersen et al., (2005), establecieron que el comportamiento se asocia con la zona destinada al pastoreo, la disponibilidad de forraje y la carga animal utilizada. Ellos estimaron una frecuencia de alimentación $18 \%$ más alta con carga animal baja (5 cerdos en $100 \mathrm{~m}^{2}$ ) en comparación con una carga alta ( 5 cerdos en $50 \mathrm{~m}^{2}$ ), siendo mayores el hozado y el comportamiento agresivo. Otras actividades naturales de los cerdos en el sistema a campo, tienen que ver con mordisquear objetos, el hozado y el pastoreo, las cuales se constituyen en la expresión de hábitos exploratorios naturales en los cerdos. Micklich et al., (1999) establecieron que los comportamientos de hozado y pastoreo en los cerdos están orientados a la búsqueda de alimento y permiten a los animales evaluar el potencial de uso de las diferentes áreas en los potreros. Estos autores indicaron también que un incremento en alguna de estas actividades puede estar motivado por la necesidad de cubrir las demandas nutricionales de los animales.

Studnitz et al., (2007) plantearon que, aunque el cerdo explora su ambiente en busca de alimentos y otros recursos para suplir sus necesidades inmediatas, también lo hace por curiosidad, para explorar nuevas características dentro de su medio ambiente y reconocer cambios ambientales. Adicionalmente, la conducta exploratoria se manifiesta mediante el hozado, olfateo, mordiendo y masticando objetos indigeribles y digeribles. Estos autores establecieron que la fabricación del nido es una modificación de esta conducta, siendo este un patrón predefinido, una acción de comportamiento heredada, que se expresa en machos y hembras. 
Guy et al., (2002) reportaron que lechones en lotes a campo durante los primeros días pasan la mayor parte del tiempo dentro del refugio, y cuando no están él, están hozando, revolcándose y explorando los lotes. También se observó que lechones nacidos al aire libre a partir de los 15 días de edad, pasaron menos tiempo en contacto con la cerda y se dedicaron a edad temprana al hozado y masticación de plantas y paja disponible. Esta actividad ejerce un efecto positivo sobre la adaptación de los lechones a la alimentación sólida, facilitando el destete. Además, esta disminución en el tiempo e intensidad del contacto entre la cerda y sus crías hace que paulatinamente disminuya la producción láctea, incrementando el consumo de alimento sólido por los lechones, lo cual disminuye el estrés posdestete.

Con relación al comportamiento de las cerdas de cría a campo, se ha establecido que sus hábitos son diurnos, después de la puesta del sol, las cerdas mantenidas en exterior reducen sus actividades y permanecen acostadas durante la noche. Este comportamiento en parte heredado, también se ve afectado por el entorno y la experiencia de cada animal, también las cerdas desarrollan comportamiento maternal a medida que avanzan en su reproducción, pero esto se ve afectado por el medio ambiente (Thodberg et al., 2002). Sin embargo, Damm et al., (2003) encontraron que las cerdas a campo tuvieron un comportamiento variado durante la construcción del nido, frecuencia cardiaca más baja en la ultima hora antes del parto, que las cerdas confinadas, lo cual permitió partos más rápidos y con menor incidencia de mortinatos. Previo al momento del parto, entre 6 y 12 horas antes del mismo, las cerdas inician la elaboración del nido, acopiando forraje, paja y palos sobre una depresión del suelo. La cantidad de material para el nido y los rasgos propios en su construcción, depende de las condiciones climáticas, pero puede ser muy variable; se han observado nidos con $20 \mathrm{~kg}$ de material y de 1 metro de alto.

Las manifestaciones naturales durante periparto incluyen la disminución en el tiempo del parto, lo cual ejerce efectos benéficos sobre la supervivencia de los lechones (Oliveiro et al., 2010). Los partos breves liberan más oxitocina y con ello la producción de calostro, incrementando la inmunidad y vitalidad de los neonatos 
siendo las cerdas más activas, pero dentro de la primera o segunda hora después del inicio del parto se hacen muy pasivas, exhibiendo una baja actividad, en decúbito lateral y no responden a los lechones. Esta reacción es considerada como estrategia evolutiva que reduce el riesgo de aplastamiento de los lechones durante las dos primeras horas posparto (Pedersen et al., 2003).

Blumetto et al., (2013) establecieron que los sistemas de producción a campo abierto evidencian menos problemas de salud, un comportamiento productivo aceptable y mayor calidad de la carne. Además los cerdos son más activos; mientras que los confinados son más sedentarios y tienen un patrón de comportamiento estable a lo largo del día. Sin embargo, el éxito de los sistemas de cerdos al aire libre puede depender de los detalles del diseño de la granja, el manejo productivo y la ubicación incluyendo el tipo de suelo y las condiciones climáticas.

Este trabajo muestra un análisis del comportamiento de cerdos bajo el sistema a campo abierto en las dos temporadas del año (seca y lluvias), donde el objetivo fue establecer información sobre el comportamiento animal en un sistema de cría de cerdos a campo abierto en el piedemonte llanero.

\section{METODOLOGÍA}

Este proyecto se desarrolló en la Unidad de Producción de Cerdos de la Universidad de los Llanos (latitud 4 04' 30.93" N, longitud 73³4' 55.78” O), ubicada en la vereda Barcelona del municipio de Villavicencio, departamento del Meta, a una altitud de $423 \mathrm{msnm}$, temperatura promedio de $27^{\circ} \mathrm{C}$, precipitación de $4050 \mathrm{~mm}$, humedad relativa del $80 \%$ correspondiente a la zona de vida de bosque muy húmedo tropical (bmh-T) (Silva et al., 2013).

Se evaluó el comportamiento de seis cerdas de cría (mestizas Landrace $\mathrm{x}$ Pietrain), de edad aproximada entre 7 y 9 meses, nulíparas que parieron por primera vez en el proceso del experimento; se hicieron las evaluaciones en dos temporadas del año: seca (Diciembre a Febrero) y lluvias (Marzo a Mayo y Agosto a Octubre), teniendo en cuenta factores como la distribución del área por los 
animales para actividades como el descanso, refugio, hozado, alimentación y consumo de agua. Además se registró el consumo de especies respecto a la oferta forrajera presente en los lotes. El proyecto tuvo una duración de 12 meses, cuyo protocolo de observación planteado fue el siguiente:

Primera fase. Establecimiento del área de observación donde se ubicaron los animales adecuando un refugio colectivo estático elaborado con ángulos metálicos y teja de zinc, con un área de $38 \mathrm{~m}^{2}$, que estaba dotado con comederos individuales y bebederos automáticos. El área estuvo definida para uso en pastoreo con tiempos de 30 días de ocupación y 30 de descanso, encerrado con cerca eléctrica, utilizándose una densidad de $450 \mathrm{~m}^{2} / \mathrm{cerda}$, mantenidas en pastoreo con gramíneas

Segunda fase. En el monitoreo de las cerdas se observó adaptabilidad, reconocimiento, comportamiento individual y grupal, respuesta frente a estímulos de peligro o amenaza, selección de alimento, horas y áreas de descanso, hozado, y zonas sucias o áreas de excreción. También se estuvo pendiente del comportamiento maternal, con el fin de determinar características etológicas en el sistema a campo abierto.

Este proceso se realizó en las dos temporadas del año con el fin de comparar cambios en el comportamiento y su expresión con los índices productivos; también se registraron los datos de temperaturas máximas y mínimas a las 7:00 a.m., 12:00 m y 4:00 p.m., con termómetro de mercurio ubicado en la zona experimental, al igual que las precipitaciones registradas con un pluviómetro por periodos de 30 días, tanto en época de lluvias como en la seca; con el fin de establecer el efecto de estas sobre el comportamiento animal.

La alimentación de los animales se basó en una dieta elaborada en la granja, acorde al estado fisiológico de los animales. Se utilizó aceite crudo de palma como base energética y torta de soya como base proteica (Tabla1). El alimento fue suministrado de manera restringida en dos raciones al día (7:30 a.m. y 4:30 p.m.), $2 \mathrm{~kg} / \mathrm{día}$ a cerdas gestantes, y en lactancia $2 \mathrm{~kg} /$ día más $200 \mathrm{~g}$ por lechón. El 
manejo reproductivo se fundamentó en el uso de un reproductor comercial (Landrace $x$ Pietran), realizando el servicio una vez se identificaba el celo.

Tabla 1. Materias primas y proporciones de la dieta suministrada a las cerdas

\begin{tabular}{ccc}
\hline Materia prima & $\%$ & Aporte de Nutrientes \\
\hline Torta de soya (molida) & 25.00 & Proteína \\
Torta de palmiste & 32.50 & Energía \\
Harina de Arroz & 19.00 & Lisina \\
Aceite de palma & 17.00 & Energía \\
Tricalfos & 5.00 & Calcio \\
Carbonato de calcio & 1.00 & Fósforo \\
Premezcla & 0.30 & Minerales y vitaminas \\
Sal blanca & 0.20 & \\
Total & $\mathbf{1 0 0 . 0}$ & \\
\hline
\end{tabular}

Fuente: Ocampo, (2002).

Cerca al momento del parto (hasta 8 días antes) las hembras fueron trasladadas a corrales de parto, los cuales estaban dotados de bebederos y comederos independientes para la cerda y sus crías; cada grupo permaneció en el corral hasta el destete (25-32 días), tiempo en el cual las cerdas volvieron a los lotes de pastoreo, de ahí los lechones pasaron a corrales de refugios colectivos donde se incluyeron en programas de levante y engorde.

\section{Registro de la información}

Como se indicó se realizaron monitoreos continuos con el fin de identificar las conductas manifestadas por las cerdas bajo este sistema durante 12 meses, haciendo observaciones desde las 07:00 am. hasta las 05:00 pm. (observación directa a los animales a intervalos de 5 minutos, repitiendo el proceso cada 15 minutos), determinando la actividad de las cerdas cuando estas se encontraran comiendo, bebiendo, pastoreando, descansando, hozando, peleando, bañándose, caminando o explorando, e interactuando, para establecer el impacto de las condiciones ambientales sobre estas actividades (Tabla 2). También se 
determinaron las zonas utilizadas: limpias, húmedas y sucias, según el comportamiento de las cerdas.

Tabla 2. Descripción de los comportamientos observados

\begin{tabular}{|c|c|c|}
\hline \multicolumn{2}{|c|}{ Comportamiento } & Descripción \\
\hline $\begin{array}{c}\text { Comer } \\
\text { concentrado }\end{array}$ & $\mathrm{C}$ & $\begin{array}{c}\text { Cerda con la cabeza dentro del comedero o } \\
\text { consumiendo concentrado }\end{array}$ \\
\hline Consumo de agua & CA & Cerdas con su hocico en el bebedero de nipple. \\
\hline Pastorear & PA & $\begin{array}{c}\text { Cerda con la cabeza entre la vegetación o masticando } \\
\text { forraje. }\end{array}$ \\
\hline Exploración & $\mathrm{E}$ & Cerdas caminando por el terreno, hozando \\
\hline Descansar & D & $\begin{array}{l}\text { Cerdas con el cuerpo en decúbito esternal o lateral, o } \\
\text { sentado sin actitud de exploración. }\end{array}$ \\
\hline $\begin{array}{l}\text { Baño en la } \\
\text { Piscina* }^{*}\end{array}$ & $\mathrm{BP}$ & $\begin{array}{c}\text { Cerdas con el cuerpo sumergido en las piscinas } \\
\text { descansando u hozando }\end{array}$ \\
\hline Hozar & $\mathrm{H}$ & $\begin{array}{c}\text { Cerda con la cabeza en el suelo desnudo revolcando el } \\
\text { suelo. }\end{array}$ \\
\hline Pelear & $P$ & Cerdas interactuando negativamente con otras cerdas. \\
\hline $\begin{array}{l}\text { Curiosear } \\
\text { humanos }\end{array}$ & $\mathrm{Ch}$ & $\begin{array}{c}\text { Cerdas desplazándose, persiguiendo y/o observando a } \\
\text { las personas que se acercan a los potreros. }\end{array}$ \\
\hline
\end{tabular}

Fuente: Adaptado de Blumetto et al., (2013). *Durante la fase de adaptación de las cerdas se construyeron depósitos de agua aprovechando los drenajes naturales de los lotes, para su uso en actividades de termorregulación, comportamiento incluido en el análisis.

Para establecer la diversidad vegetal del campo utilizado por las cerdas y nivel de cobertura de las especies presentes, se realizaron transeptos y aforos por metro cuadrado para determinar el número de especies presentes y el grado de dominancia de las mismas. Los muestreos se realizaron simultáneamente con las de suelos, al inicio del periodo experimental y después cada 3 meses.

La información registrada fue analizada en IBM SPSS Statistic (IBM, 2011), versión 20; las variables fueron evaluadas para distribución normal, pruebas de Levene para homogeneidad de varianzas, prueba de $t$-Student para variables independientes; estas fueron el consumo de agua, pastoreo exploración, descanso, bañarse en la piscina, hozar, pelear y curiosear con humanos. 


\section{RESULTADOS Y DISCUSIÓN}

Las acciones de consumo de agua, pastoreo exploración, descanso, bañarse en la piscina y pelear, fueron altamente influenciadas por la época del año $(P<0.001)$ (Tabla 3), También se evidenciaron cambios en las actividades de los lotes en relación con los cambios de temperatura ambiental registrados.

Tabla 3. Actividades desarrolladas y el tiempo (minutos) por las cerdas de cría en observaciones realizadas de 7:00 am a 5:00 pm.

\begin{tabular}{cccc}
\hline Actividad & Época* $^{*}$ & $\begin{array}{c}\text { Media } \pm \text { Desviación } \\
\text { Estándar }\end{array}$ & Valor de P \\
\hline \multirow{2}{*}{ Consumo de concentrado } & Lluvias & $11.81 \pm 1.65$ & NS \\
& Seca & $11.03 \pm 2.87$ & \\
Consumo de agua & Lluvias & $51.05 \pm 5.07$ & 0.001 \\
Pastoreo & Seca & $115.98 \pm 10.81$ & \\
& Lluvias & $130.0 .7 \pm 10.74$ & 0.001 \\
Exploración & Seca & $118.98 \pm 11.16$ & \\
& Lluvias & $16.10 \pm 1.64$ & 0.001 \\
Descanso & Seca & $23.8 .49 \pm 4.23$ & \\
& Lluvias & $309.27 \pm 10.20$ & 0.001 \\
Baño en Piscina & Seca & $185.49 \pm 8.69$ & \\
& Lluvias & $51.15 \pm 5.07$ & 0.001 \\
Hozar & Seca & $115.98 \pm 10.81$ & \\
& Lluvias & $14.31 \pm 2.43$ & NS \\
Pelear & Seca & $14.90 \pm 4.62$ & \\
& Lluvias & $5.96 \pm 1.22$ & 0.001 \\
Curiosear Humanos & Seca & $14.90 \pm 0.4 .62$ & \\
& Lluvias & $10.38 \pm 1.86$ & 0.012 \\
\hline
\end{tabular}

*Precipitación pluviométrica acumulada para la época de lluvias $727 \mathrm{~mm}$ y en verano $54 \mathrm{~mm}$

Respecto a las actividades realizadas por las cerdas durante un día normal de actividad (10 horas) (Figuras 1 y 2), bajo el sistema de cría a campo abierto según la temporada del año (seca o lluvias) en la densidad aplicada ( $450 \mathrm{~m}^{2} /$ animal), se 
observó que la dedicación de tiempo a bañarse en la piscina y al consumo de agua, fue influenciado indudablemente por la época del año $(P<0.001)$ (Tabla 3$)$. En temporada seca, las cerdas dedicaron $36 \%$ de tiempo a estas actividades en comparación con la época de lluvias que solo fue el 12\% (Gráficas 1 y 2). Igualmente, las estrategias de termorregulación utilizadas a lo largo del día fueron influenciadas por los cambios de temperatura, donde se evidenció la disponibilidad de alternativas para dicha actividad como la presencia de vegetación y refugios; las cerdas prefirieron el uso de las piscinas en las horas de altas temperaturas (11:00 am. a 3:00 pm.). Este comportamiento fue más prolongado en la temporada seca, mientras que en época lluvias se incrementó el tiempo de descanso en los refugios (47\%), en comparación con $29 \%$ en la época seca (Gráficas 1 y 2).

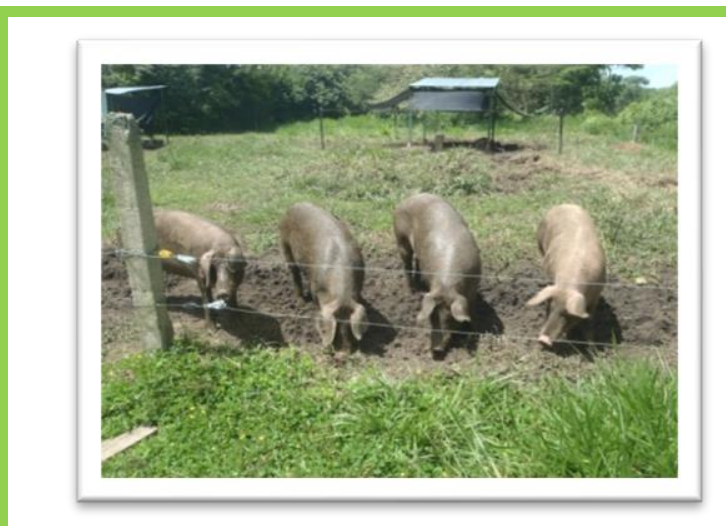

Figura 1. Actividades de las cerdas

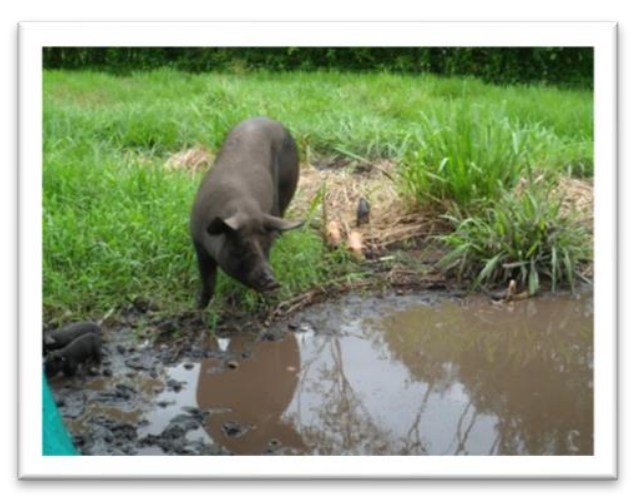

Figura 2. Piscinas naturales

El hozado, fue la actividad que se incrementó en puntos focales del campo, generando depresiones de profundidad que, por acción de las pendientes y patrones de drenaje en diversos sitios, actuaron como depósito de agua en la época de lluvias, los cuales fueron denominados "piscinas" siendo usados como estrategia de termorregulación por las cerdas tanto en temporada seca como de lluvias. La observación directa permitió establecer el alto impacto de estas piscinas sobre el comportamiento de las cerdas, puesto que dedicaron $115.98 \pm 10.81$ minutos para refrescarse durante la época seca, lo cual equivale a un $18 \%$ del tiempo utilizado para esta acción en un día de observación (10 horas) (Gráfica 1 y Tabla 3). 

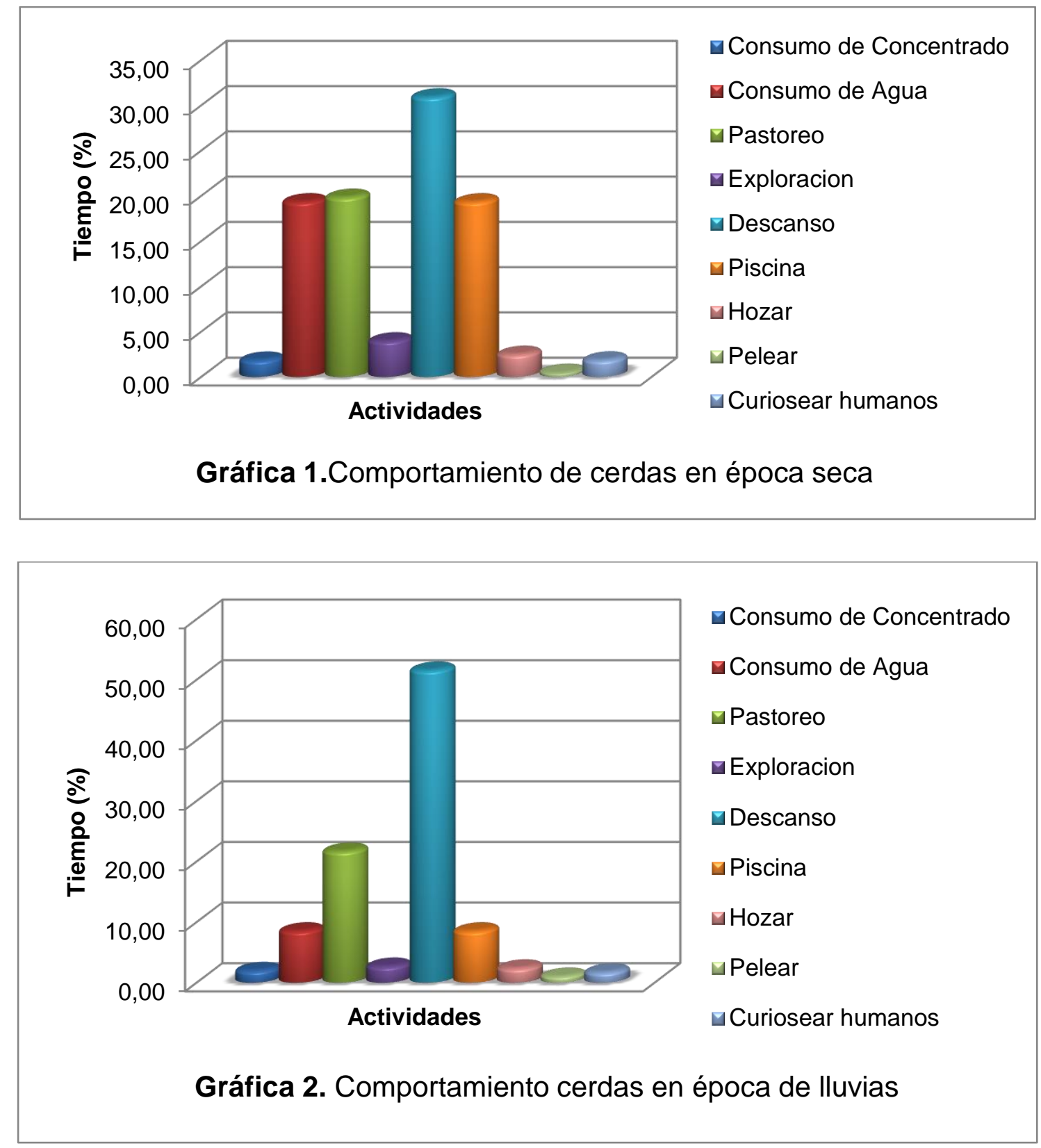

Se observaron cambios de comportamiento en las temperaturas máximas y mínimas entre épocas $(P<0.001)$, las alteraciones en las actividades de termorregulación, bañarse o descansar, exploración, pastoreo y hozar pueden ser atribuidas a los cambios de temperatura ambiental durante del día; por otra parte, el incremento de las precipitaciones, conllevó a la disminución del tiempo dedicado a la exploración. Se puede notar, que las cerdas permanecieron mayor tiempo en la piscina durante la época seca, cuando las temperaturas promedio fueron de $36.6^{\circ} \mathrm{C}$, los animales utilizaron este mecanismo para reducir el estrés calórico, 
según el tiempo dedicado a dicha actividad fue de $115.98 \pm 10.81$ minutos $(20 \%$ del tiempo del día dedicado a esta actividad). En cuanto a la temporada de lluvias, el descanso en los refugios fue la actividad que mayor demanda tuvo durante el día (309.27 \pm 10.20 minutos) lo que equivale al $53 \%$ del tiempo del día dedicado a esta acción. Las altas precipitaciones (16.52 mm/día) en temporada de lluvias, acompañada de tormentas eléctricas, representaba algún tipo de peligro para los animales, optando por esperar para consumir el concentrado de la ración de las 4:30 pm., lo cual ocasiono reducción en las demás actividades, exceptuado la de descansar en el refugio (Gráficos 1 y 2, Tabla 3). Se evidenció que la distribución de las actividades de los animales se ve afectada por diferentes estímulos, principalmente la presencia de humanos, posiblemente porque es interpretada como suministro de alimento, por lo cual las cerdas dejan de exhibir el comportamiento natural y lo sustituyen por expresiones de curiosidad, desplazándose hasta la persona y realizando algunos ruidos característicos. El uso de las áreas está determinado por las condiciones climáticas, encontrándose mayor uso de los sitios que tenían alternativas de termorregulación, sombra natural o piscinas.

La identificación de las especies vegetales presentes en el lote permitió establecer una mezcla de gramíneas nativas, leguminosas y arvenses; donde dominaba el pasto estrella (Cynodon nlemfuensis) principalmente en partes bien drenadas; se estableció una clara preferencia por el consumo de arvenses tales como Mimosa púdica, Senna covanens Vss, Sida spinosa, Desmodium spp, entre otras y brotes de gramíneas como Brachiaria decumbens, Brachiaria humidicola y Cynodon nlenfluensis; otra leguminosa encontrada en los lotes fue Arachis pintoi la cual fue consumida en preferencia por la cerdas. Pese a que el consumo de forraje se tuvo en cuenta como patrón de comportamiento, no fue considerado desde el punto de vista nutricional de acuerdo con Edwards, (2003). El tiempo dedicado a esta actividad para la época lluviosa y seca se redujo (118.98 \pm 1161 Vs $30.07 \pm 10.74$ minutos), reflejándose el impacto que tuvo el ambiente sobre el comportamiento de los animales (Gráficas 1 y 2, Tabla 3). 
De manera rutinaria los animales después de alimentarse buscan la zona sucia del lote para depositar sus excretas, esta actividad va seguida de la búsqueda de un sitio apropiado para descansar, cuando el sitio está ocupado pueden dedicarse al hozado. Previo al momento del descanso, los animales tienden a socializar entre sí, mediante el juego y contacto entre ellos. Se observó que las cerdas tienden a escoger algunos sitios específicos para sus actividades (alimentarse, descansar). A medida que los animales crecían la competencia por el alimento se hacía más fuerte y de una manera más agresiva, notándose jerarquía a la hora de recibir el alimento, pero cabe resaltar que los animales dedicaron mayor tiempo al descanso, comprobando que el manejo en este tipo de producción ofrece un adecuado confort a los animales y en consecuencia un mayor bienestar animal. La constante incorporación de excretas, orina y efluentes a lo largo del periodo de observación, y el posterior pastoreo ejercido por los animales, terminaron convirtiéndose en abono, enriqueciendo el suelo y así logrando posiblemente mayor diversidad vegetal del lote donde se encontraban refugiadas.

\section{Estación y partos}

Las cerdas gestantes fueron observadas diariamente por 2.5 horas, a intervalos de 15 minutos, el monitoreo permitió establecer cambios en la expresión de las actividades y ubicación de los animales en función de los cambios en la temperatura a lo largo del día, de acuerdo a Geonaga, (2010). Las cerdas fueron preñadas por monta directa; respecto a su cambio de actividades, las cerdas se apartan del grupo en promedio 2-3 días previos al parto, donde buscan cualquier tipo de material (ramas o pasto) para la elaboración del nido, buscando zonas limpias, bien drenadas y fuera de cualquier posible predador; en las cerdas primerizas las labores del parto implicaron mayor trabajo, viéndose esto reflejado en muertes de los lechones por aplastamiento.

Por otra parte, el monitoreo de las temperaturas máximas y mínimas a lo largo del día (10 horas) permitieron establecer cambios en la expresión de las actividades y ubicación de los animales según la temperatura, la cual fue similar en cada época $(P>0.05)$, para época seca fluctuó entre 28.1 y $39.1^{\circ} \mathrm{C}$, y para la temporada de 
lluvia entre 20.5 y $24.9^{\circ} \mathrm{C}$. Aunque las observaciones realizadas evidenciaron picos marcados de actividad durante el día, las cerdas exhibieron mayores actividades de exploración en los intervalos 8:00-10:30 a.m. y 3:45-5:00 p.m.; el uso de las piscinas es continuo y más evidente en el intervalo 10:45 a.m.-3:30 p.m., los refugios son utilizados en mayor magnitud en el intervalo de 1:00-3:00 p.m. Pese a que tanto en las horas de la mañana como en la tarde las cerdas realizan todas las actividades descritas, es más intensa su expresión en horas de la mañana, esto podría estar definido por las temperaturas promedio que tanto para la temporada seca como de lluvias, fueron menores en las mañanas. La mayor expresión de actividades exploratorias se registró en la mañana después del suministro del alimento; otro pico de actividad de menor intensidad fue observado en la tarde, también posterior al suministro de alimento. Este comportamiento puede estar relacionado con el patrón natural de comportamiento de los cerdos (Wulbers et al., 1990).

Con base en las temperaturas registradas se puede deducir que estas si influyeron en el comportamiento o actividad del animal puesto que el nivel de temperatura aumenta o disminuye los periodos de descanso o refugio bajo techo, de manera similar como ocurre con los mecanismos de termorregulación; los animales no se exponen a altas temperaturas o caso contrario cuando se registran fuertes lluvias, puesto que las cerdas prefieren esperar que la lluvia disminuya para así continuar con sus actividades; en condiciones extremas los animales prefieren resguardarse bajo techo y esperar que dicha condición empiece a descender. Al igual estos cambios influyen sobre la calidad y cantidad de forraje, lo que cambia sus actividades de pastoreo u hozado, disminuyendo o aumentando así los minutos dedicados a estas actividades por parte del animal durante el día.

Mediante la observación realizada a las cerdas establecidas en un sistema a campo abierto se pudo notar en gran medida conductas naturales propias de la especie, como la creación de un nido al momento del parto (Figura 3), o el establecimiento de zonas específicas diferenciándolas en sucias, limpias, de reposo, características que están implícitas en las cerdas, aun cuando estas no 
han tenido contacto alguno con el campo o el medio ambiente. No se evidencio comportamientos estereotipados o conductas particulares que indicaran algún tipo de estrés como se pueden presentar en una explotación de tipo confinamiento.

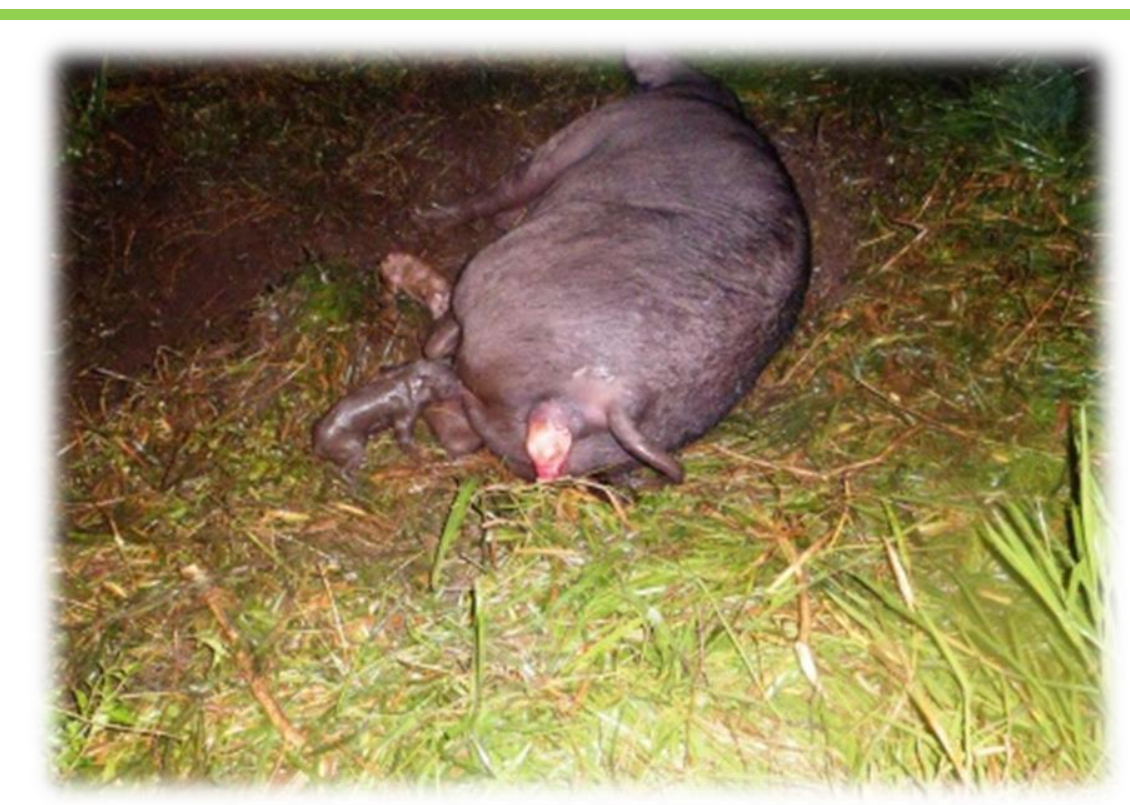

Figura 3. Registro de un parto bajo el sistema a campo abierto

Las conductas o actividades para las dos temporadas del año, fueron diferentes para las variables consumo de agua, pastoreo exploración, descanso y baño en piscina, lo cual influyó en el tiempo de refugio bajo techo, evidenciando diferencias en el tiempo dedicado a las piscinas el cual fue mayor para la época seca como era de esperar; durante las dos épocas se mantuvieron las zonas establecidas por los propios animales. Los animales definieron una tendencia de comportamiento: consumo de alimento, seguido de búsqueda de la zona sucia para depositar sus excretas y posteriormente el descanso, siempre en sitios específicos, los cuales se mantuvieron durante todo el periodo.

\section{CONCLUSIONES}

El comportamiento de las cerdas sugiere una interesante capacidad de adaptación

a las condiciones de temperatura, precipitación y características del terreno (drenaje), así como al sistema de manejo, puesto que el sistema a campo abierto 
permite expresar conductas naturales, mitigando comportamientos estereotipados, de ocio y peleas por jerarquía, debido a que el microambiente en el que se encuentran los animales simula que se encuentren en libertad, dando al animal la posibilidad de expresar todo su potencial en cuanto al comportamiento social, reproductivo, maternal e individual (hozar, explorar, comer y beber).

\section{RECOMENDACIONES}

Se requiere profundizar sobre el comportamiento animal en las condiciones de la Orinoquía y su dinámica de precipitación, radiación solar y temperatura ambiental para el diseño de instalaciones apropiadas que garanticen condiciones de confort y estrategias de manejo.

\section{REFERENCIAS BIBLIOGRÁFICAS}

1. Andersen IL, Berg S, Boe K. Crushing of piglets by the mother sow (Sus scofa) - Purely accidental or a poor mother? Applied Animal Behaviour, 93 (3): 229243. 2005.

2. Blumetto OR, Calvet $S$, Estellés F, Villagrá A. Comparison of extensive and intensive pig production systems in Uruguay in terms of ethologic, physiologic and meat quality parameters. Revista Brasileira de Zootecnia, 42 (7): 521-529. 2013.

3. Campiño GE, Ocampo Á. Comportamiento de cerdos de engorde en un sistema de cama profunda utilizando racimos vacíos de palma de aceite. Revista Orinoquia, 14 (2): 147-159. 2010.

4. Cardozo AF, Rodríguez LE. Potencial y necesidades de investigación sobre el cerdo criollo en los llanos de Colombia y Venezuela. Revista Computadorizada de Producción Porcina, 17 (2): 107-115. 2010.

5. Damm BI, Lisborg L, Vestergaard KS, Vanicek J. Nest-building, behavioural disturbances and heart rate in farrowing sows kept in crates and Schmid pens. Livestock Production Science, 80 (3): 175-187. 2002.

6. Edwards SA. Intake of nutrients from pasture by pigs. Proceeding of the Nutrition Society, 62 (2): 257-265. 2003.

7. Goenaga P. Comportamiento materno de la cerda y sus implicancias en la producción. Actualización bibliográfica. Sitio Argentino de Producción Animal. $2010 . \quad$ Disponible En: http://www.produccionanimal.com.ar/produccion porcina/00-produccion porcina general/154Comportamiento materno.pdf

8. Gomero L, Velásquez H (Ed). Manejo ecológico de suelos. Conceptos, experiencias y técnicas. Red de Acción en Alternativas al uso de Agroquímicos. Urb. Las Brisas, Cercado, Lima, Perú, p 11-12. 1999. 
9. Guy J, Rowlinson P, Chadwick JP, Ellis M. Behaviour of two genotypes of growing-finishing pig in three different housing systems. Applied Animal Behaviour Science, 75 (3): 193-206. 2002.

10. IBM Corp. Released. IBM SPSS Statistics for Windows, Version 20.0. Armonk, NY: IBM Corp. 2011.

11. Micklich D, Matthes HD. Die weidehaltung von sauen verschiedener rassen auf flußauenstandorten. Arch. Tierz., Dummerstorf, 42 (2): 161-173. 1999.

12. Muñoz L. Bienestar de los cerdos: Las normas europeas y una propuesta de bienestar razonable. En: Congreso Latinoamericano de Suinocultura. Foz de Iguazú. p 45-52. 2002.

13. Muriel E, Ruiz J, Ventanas J, Antequera T. Free-range rearing increases (n-3) polyunsaturated fatty acids of neutral and polar lipids in swine muscles. Food Chemistry, 78 (2): 219-225. 2002.

14. Oliveiro C, Heinonen M, Valros A, Peltoniemi O. Environmental and sowrelated factors affecting the duration of farrowing. Animal Reproduction Science, 119 (1-2): 85-91. 2010.

15. Ocampo A. High lipid diets based on oil palm for growing-fattening pigs. PhD Thesis. University of London, Imperial College, Department of Agricultural Sciencies. 352 p. 2002.

16. Pedersen L, Damm BI, Marchant JN, Jensen KH. Effects of feed-back from the nest on maternal responsiveness and postural changes in primiparous sows during the first $24 \mathrm{~h}$ after farrowing onset. Applied Animal Behaviour Science, 83 (2): 109-124. 2003.

17. Rodarte LP. Comportamiento, manejo y bienestar del cerdo. Departamento de Etología y Fauna Silvestre. Facultad de Medicina Veterinaria y Zootecnia. Universidad Nacional Autónoma de México. p 7-9. 2001. Disponible En: http://amaltea.fmvz.unam.mx/ETOLOGIA/TEMAS/CERDOS/COMPORTAMIE NTO,\%20MANEJO\%20Y\%20BIENESTAR\%20CERDO.pdf

18. Rudine AC, Sutherland MA, Hulbert L, Morrow JL, McGlone JJ. Diverse production system and social status effects on pig inmunity and behavior. Livestock science, 111 (2): 86-95. 2007.

19. Sarria P, Pérez H, Silva J. Caracterización de las actividades de comportamiento de cerdos al aire libre. Livestock Research for Rural Development, 13 (4): Art 34. 2001. Disponible En: http://www.Irrd.org//rrd13/4/sarr134.htm

20. Silva AC, Zuluaga AM., Roa ML. Evaluación de la utilización de Cratylia argentea como suplemento en dietas para pollos de engorde. Rev. Sist. de Prod. Agroecol, 4 (1): 140-152. 2013.

21. Studnitz M, Jensen MB, Pedersen LJ. Why do pigs root and in what will they root?: A review on the exploratory behaviour of pigs in relation to environmental enrichment. Applied Animal Behaviour Science, 107 (3-4): 183197. 2007.

22. Thodberg K, Jensen K, Herskin M. Nursing behavior, postpartum activity and reactivity in sows: Effects of farrowing environment, previous experience and temperament. Applied Animal Behaviour Science, 77(1): 53-76. 2002.

23. Wood D, Jensen $P$, Algers B. Behaviour of pigs in a novel semi-natural environment. Biology of Behaviour, 15 (2): 62-73. 1990. 